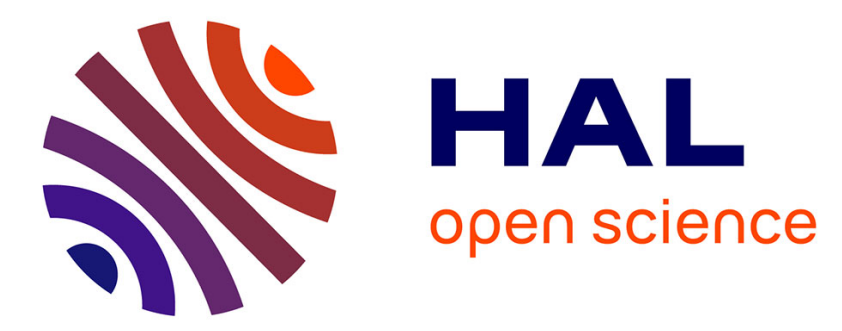

\title{
Photo-Acoustic Tomography (PAT) based on Laser Optical Feedback Imaging (LOFI) of surface displacements
}

\author{
Vadim Girardeau, Olivier Jacquin, Olivier Hugon, Bathilde Riviere, \\ Boudewijn van Der Sanden, Eric Lacot
}

\section{To cite this version:}

Vadim Girardeau, Olivier Jacquin, Olivier Hugon, Bathilde Riviere, Boudewijn van Der Sanden, et al.. Photo-Acoustic Tomography (PAT) based on Laser Optical Feedback Imaging (LOFI) of surface displacements. Applied optics, 2019, 58 (26), pp.7195-7204. 10.1364/AO.58.007195 . hal-02282968

\section{HAL Id: hal-02282968 \\ https://hal.science/hal-02282968}

Submitted on 10 Sep 2019

HAL is a multi-disciplinary open access archive for the deposit and dissemination of scientific research documents, whether they are published or not. The documents may come from teaching and research institutions in France or abroad, or from public or private research centers.
L'archive ouverte pluridisciplinaire HAL, est destinée au dépôt et à la diffusion de documents scientifiques de niveau recherche, publiés ou non, émanant des établissements d'enseignement et de recherche français ou étrangers, des laboratoires publics ou privés. 


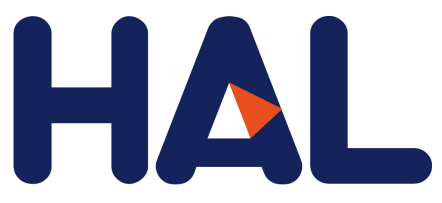

archives-ouvertes

\title{
Photo-acoustic tomography based on laser optical feedback imaging of surface displacements
}

\author{
Vadim Girardeau, Olivier Jacquin, Olivier Hugon, Bathilde Riviere, \\ Boudewijn van der Sanden, Eric Lacot
}

\section{To cite this version:}

Vadim Girardeau, Olivier Jacquin, Olivier Hugon, Bathilde Riviere, Boudewijn van der Sanden, et al.. Photo-acoustic tomography based on laser optical feedback imaging of surface displacements. Applied optics, Optical Society of America, 2019, 58 (26), pp.7195. 10.1364/AO.58.007195 . hal-02282968

\section{HAL Id: hal-02282968 \\ https://hal.archives-ouvertes.fr/hal-02282968}

Submitted on 10 Sep 2019

HAL is a multi-disciplinary open access archive for the deposit and dissemination of scientific research documents, whether they are published or not. The documents may come from teaching and research institutions in France or abroad, or from public or private research centers.
L'archive ouverte pluridisciplinaire HAL, est destinée au dépôt et à la diffusion de documents scientifiques de niveau recherche, publiés ou non, émanant des établissements d'enseignement et de recherche français ou étrangers, des laboratoires publics ou privés. 


\title{
Photo-Acoustic Tomography (PAT) based on Laser Optical Feedback Imaging (LOFI) of surface displacements
}

\author{
Vadim Girardeau, ${ }^{1}$ Olivier Jacquin, ${ }^{1}$ Olivier Hugon, ${ }^{1}$ Bathilde Riviere ${ }^{1}$ \\ BOUDEWIJN VAN DER SANDEN, ${ }^{2}$ AND ERIC LACOT ${ }^{1, *}$ \\ ${ }^{1}$ Univ. Grenoble Alpes, CNRS, LIPhy, F-38000 Grenoble, France \\ ${ }^{2}$ Univ. Grenoble Alpes, INSERM, CNRS, TIMC-IMAG, F-38000 Grenoble, France \\ *Corresponding author: eric.lacot@univ-grenoble-alpes.fr
}

Received XX Month XXXX; revised XX Month, XXXX; accepted XX Month XXXX; posted XX Month XXXX (Doc. ID XXXXX); published XX Month XXXX

We present how a Laser Optical Feedback Imaging (LOFI) setup can be used for the optical detection of ultrasound in Photo-Acoustic Tomography (PAT). A PAT image is reconstructed by an inversion algorithm using surface displacement measurements made at several locations with our LOFI setup and following the optical irradiation with a pulsed Nd:YAG laser of a sample with absorbing inclusions. The width of the reconstructed inclusions and the SNR of the reconstructed images are firstly studied on the numerical model of a sample with 3 absorbing inclusions (i.e. with 3 acoustic punctual sources). Finally an experimental PAT image of a phantom composed of two polyamide tubes with an internal diameter of $800 \mu \mathrm{m}$ filled with red ink and submerged at $\mathbf{- 3 . 5} \mathbf{m m}$ depth in a tank filled with water is reconstructed. Experimentally, the water surface displacement measurements has been made with our LOFI vibrometer which provides an amplitude sensitivity of $1 \mathrm{~nm}$ (for a single-shot measurement) in a detection bandwidth of roughly $1 \mathrm{MHz}$ adapted to the detection of the polyamide tubes. Under our experimental conditions, the surface energy densities, of the LOFI focalized beam for the detection and of the pulsed Nd:YAG laser used for the irradiation, are compatible with the MPE (Maximum Permissive Exposure) for future biomedical measurements. The SNR and the resolution of the reconstructed PAT images are in good agreement with the theoretical predictions.

\section{INTRODUCTION}

Photo-Acoustic (PA) imaging, which is based on the generation of an acoustic wave from an absorbing object being illuminated by a pulsed or a modulated laser, is a very interesting tool [1-5] in biomedical application. Indeed, PA imaging allows to combine the main advantages of the optical and ultrasound imaging methods, which are the high endogenous (or exogenous) optical absorption contrast in heterogeneous tissues and the low diffusion and absorption of ultrasound waves $\left(1 \mathrm{~dB} \cdot \mathrm{cm}^{-1} \cdot \mathrm{MHz}^{-1}\right)$ in the human body [6]. Whatever the imaging mode: OR-PAM (Optical Resolution PhotoAcoustic Microscopy), AR-PAM (Acoustic Resolution Photo-Acoustic Microscopy) or PAT (Photo-Acoustic Tomography), PA Imaging provides highly contrasted images with a ratio between the transversal resolution and the penetration depth roughly equals to $1 / 100$, e.g.: a resolution of $100 \mu \mathrm{m}$ at $1 \mathrm{~cm}$ depth or of $1 \mu \mathrm{m}$ à $100 \mu \mathrm{m}$ depth) [7]. This is the average limit of several medical imaging techniques, such as MRI, CT and ultrasound. As mentioned in [8], piezoelectric transducers (PZT) are commonly used to detect laser-induced ultrasonic waves in PA imaging. However, they typically lack adequate broadband sensitivity at high ultrasonic frequency (i.e. at high resolution) whereas their bulky size and optically opaque nature cause technical difficulties in integrating PA imaging with conventional optical imaging modalities. To overcome these limitations, optical methods of ultrasound detection were developed and shown their unique applications in PA imaging. We have [8]: the free-space optics based ultrasound detection (Michelson and Mach Zehnder interferometers), The fiber optics based ultrasound detection (fiber optics interferometer, single fiber sensor with Bragg grating or with Fabry Perrot cavity), the photonic integrated circuit detectors (polymer optical waveguide sensor, micro-cavity resonators) and the ultrasound detection via optical interface (Fresnel reflection, photonic crystal cavity, surface plasmon resonance, Metamaterials). 
The main objective of the present paper is to demonstrate how a Laser Optical Feedback Imaging (LOFI) setup (which is an optical free space technic [8]) can be used for optical detection of ultrasound in PhotoAcoustic Tomography (PAT). Nanometer measurements based on SMI (Self Mixing Interferometry) have already been realized, either for acoustic perturbations or for nm-sized vibrations [9-14]. Here, the use of a LOFI setup is motivated by the combination of the four following reasons: i) The LOFI interferometer is always self-aligned because the laser simultaneously fulfils the functions of the source (i.e. photonsemitter) and of the photo-detector (i.e. photons-receptor); ii) The phase of the interferometric signal (i.e. of the LOFI beating) is sensitive to the round-trip distance between the laser and the vibrating target. iii) The LOFI sensitivity allows analysis of targets with a weak backreflected (or back-scattered) electric field. iv) The LOFI detection is shot noise limited (even with a low power laser and a conventional detection) in a frequency range located near the relaxation oscillation frequency of the laser [15-16]. Therefore, the LOFI setup can respect medical conditions: security and easy to integrate.

This paper is organized as follows. In the first section, the principle of the acquisition of PA signal with a LOFI set-up is explained. The next section deals with principle of the image reconstruction from the surface displacement measurements. The point spread function (PSF) and the signal to noise ratio (SNR) of the reconstructed images are studied. Analytical predictions are compared with the results of a numerical simulations performed on 3 punctual acoustic sources immersed in water. Finally, an in depth experimental image reconstruction obtained from the LOFI signal is shown, where the absorbing sample, illuminated by a green pulsed laser, is a phantom composed of two polyamide tubes filled with red ink and submerged at $-3.5 \mathrm{~mm}$ depth in a tank filled with water. The final section is a general discussion of these results and possible applications in in vivo Photo-Acoustic (PA) imaging

\section{PRINCIPLE OF THE SIGNAL ACQUISITION}

A schematic diagram of the Photo-Acoustic Tomography (PAT) based on Laser Optical Feedback Imaging (LOFI) is shown in Fig. 1. Experimentally, the Photo-Acoustic (PA) effect is generated by a frequency doubled Nd:YAG laser $(\lambda=532 \mathrm{~nm})$ with a pulsed width:

$\Delta \tau_{L}=10 \mathrm{~ns}$ and a repetition rate of $10 \mathrm{~Hz}$. The laser light is send on absorbing inclusions characterized by the absorption coefficient $\mu_{a} \approx \mathrm{cm}^{-1}$ and immersed at several millimeters depth in a tank filed with water. After a time delay (linked to the sound velocity in water $c_{a}=1500 \mathrm{~m} / \mathrm{s}$ ), the transient pressure waves generated by the thermal expansion of the absorbing medium reach the air/water interface (i.e. the plane $z=0 \mathrm{~mm}$ ), which is then transiently displaced of a few nanometers.

To measure the time resolved surface displacement (induced by the PA effect), we have used a LOFI (Laser Optical Feedback Imaging) interferometer, which is a very sensitive heterodyne interferometer involving the laser dynamics [15-16]. In this imaging system, an optical beating occurs inside the laser cavity, between the intra-cavity light and the light backscattered by the target. By this way, the laser is the source and also the detector, which implies that it is very easy to use (this optical system is self-aligned) and the measures are "shot-noise" limited. The LOFI laser is a CW Nd:YAG microchip laser with a maximum available power of several $\mathrm{mW}$ at a wavelength of $\lambda_{L}=1064 \mathrm{~nm}$ and a relaxation oscillation frequency $F_{R}$ in the megahertz range. The microchip laser beam is focused on the air/water interface and part of the light reflected and/or scattered by the moving interface returns inside the laser cavity after a second pass through the frequency shifter. Therefore, the optical frequencies of the reinjected light are shifted by $F_{e}$. This frequency shift can be adjusted and is typically of the order of the laser relaxation oscillation frequency $F_{R}$ to obtain a shot noise limited signal [15-16]. The microchip laser cavity is relatively short (typically $\leq 1 \mathrm{~mm}$ ) which ensures a high damping rate of the laser cavity (typically $\approx 10^{9} s^{-1}$ ) and therefore a good sensitivity to weak optical feedback $[15,16]$.

For a given position $(x, y)$ of the laser focalizing spot on the water surface (i.e. the plane $z=0$ ), and in the case of weak optical reinjection, the coherent interaction (beating) between the lasing electric field and the frequency-shifted reinjected field leads to a sinusoidal modulation of the laser output power. For detection purposes, a small part of the laser output beam is sent to a photodiode. The delivered voltage is processed by a PC to finally obtain a quantitative measurement of the small local displacement of the air/water interface: $d_{a}(x, y, t)<<\lambda_{L}$ [14]. Experimentally, a LOFI movie of the surface displacement can be obtained pixel by pixel (i.e. point by point, line after line) by a full $2 \mathrm{D}$ galvanometric scanning of the microchip laser focal spot on the air/water interface or by a displacement of the absorbing target by using a motorized translation stage. Each LOFI punctual measurements is triggered on the optical irradiation of the sample by the pulsed Nd:YAG laser.

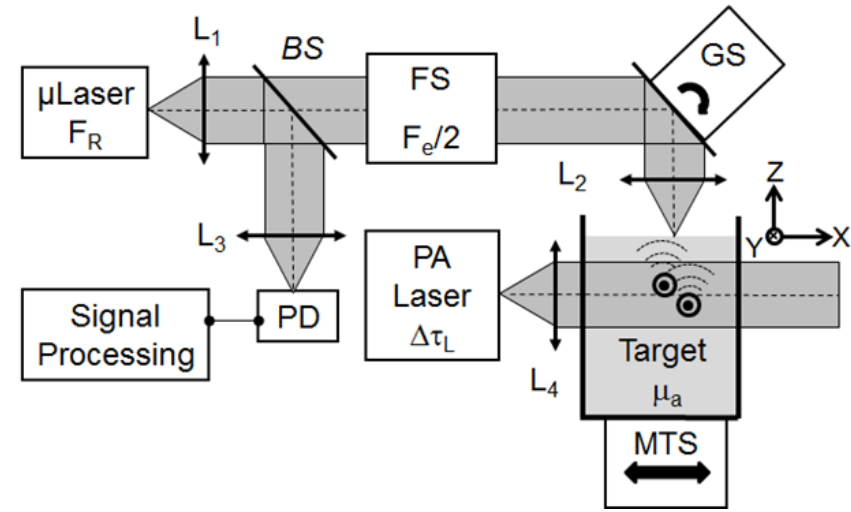

Fig. 1. Schematic diagram of PAT based on a LOFI setup. PA Laser: Laser with a pulse width $\Delta \tau_{L}$ for PA generation; Target: Two parallel polyamide tubes filled with ink with an absorption coefficient $\mu_{a}\left(\mathrm{~cm}^{-1}\right)$; $\mu$ Laser: microchip laser, with a relaxation oscillations frequency FR, for LOFI measurement of surface displacements. L1, L2, L3 and L4: Lenses, BS: Beam Splitter, PD: Photodiode, FS Frequency Shifter with a round trip frequency-shift $F_{e}$ GS: Galvanometric Scanner, MTS: Motorized translation stages.

\section{PRINCIPLE OF THE IMAGE RECONTRUCTION}

\section{A. Image reconstruction algorithm}

The image reconstruction algorithm is firstly studied on a numerical example. Fig. 2 shows the reconstruction of four 2D images obtained from the simulation of the surface temporal displacements induced by $j_{\text {max }}=3$ acoustic waves. For an easier comparison of the different images, the size of the reconstruction widows is always the same. In this numerical example, the acoustic sources are immersed in water and located on a vertical line perpendicular to the water surface and 
the time evolution of the surface displacement is obtained at $i_{\text {max }}=11$ distinct locations on the water surface.

In the present numerical simulation, the time evolution of the surface displacement at the detection locations $\left(X_{d, i}, Y_{d, i}, Z_{d, i}=0\right)$ with $i \in\left[1,2, \ldots, i_{\max }\right]$ is given by:

$$
\begin{aligned}
d_{i}(t) & =d_{a}\left(X_{d, i}, Y_{d, i}, t\right) \\
& =\sum_{j=1}^{j \max } A_{s, j}\left(\frac{S_{s, j}}{\Lambda_{s, j} r_{i, j}}\right)^{n} P_{s, j}\left(t-\tau_{i, j}\right),
\end{aligned}
$$

where for the acoustic source labeled $j \in\left[1,2, \ldots, j_{\max }\right], A_{s, j}$ is the displacement amplitude at the source location, while $S_{s, j}$ is the characteristic surface of the source. $r_{i, j}=\sqrt{\left(X_{d, i}-X_{s, j}\right)^{2}+\left(Y_{d, i}-Y_{s, j}\right)^{2}+\left(Z_{d, i}-Z_{s, j}\right)^{2}}$ is the geometrical distance between the $i_{t h}$ detection location $\left(X_{d, i}, Y_{d, i}\right.$, $\left.Z_{d, i}\right)$ and the $j_{t h}$ source location $\left(X_{s, j}, Y_{s, j}, Z_{s, j}\right)$, while $\tau_{i, j}=\frac{r_{i, j}}{c_{a}}$ is the corresponding time delay for an acoustic wave propagating with a velocity of $c_{a}=1500 \mathrm{~m} / \mathrm{s}$ in water. In Eq. (1), the exponent $n$ allows to describe different types of acoustic wave: $n=0$ for plane waves; $n=0.5$ for cylindrical waves; $n=1$ for spherical waves. Always in Eq. (1), the dimensionless temporal profile of the acoustic wave is given by: $P_{s, j}(t)=\exp \left(-2\left(\frac{t}{\Delta \tau_{s, j}}\right)^{2}\right)$, where $\Delta \tau_{s, j}$ is the time width of the detected acoustic signal generated by the photo-acoustic effect with the corresponding wavelength $\Lambda_{s, j}=c_{a} \Delta \tau_{s, j}$. Due to a convolution effect, one have: $\Delta \tau_{s, j} \approx \sqrt{\Delta \tau_{L}^{2}+\Delta \tau_{a, j}^{2}+\Delta \tau_{d}^{2}}$, where $\Delta \tau_{L}$ is the pulse width of the PA laser, $\Delta \tau_{a, j}$ are the time width of the emitted acoustic waves and $\Delta \tau_{d}$ is the time response of the detection which corresponds to a detection bandwidth of: $\Delta F_{d}=1 / \Delta \tau_{d}$.

In our numerical simulation the following simplification has been used:

i/ The 3 sources have the same size ( $S_{s, j}=S_{a}$ ), and emit spherical waves ( $n=1$ ) with the same amplitude ( $A_{s, j}=A_{a}$ ).

ii/ For the photoacoustic point of view, we have supposed that not only the thermal diffusion, but also the volume expansion of the absorber during the laser illumination period is negligible. This condition is referred to as the acoustic stress confinement $\left(\Delta \tau_{a, j}>\Delta \tau_{L}\right)$ ) [1-2]. To study the Point Spread Function (PSF) of the reconstructed image with our PAT setup, the acoustic sources need to be considered as being punctual sources $\left(\Delta \tau_{d}>\Delta \tau_{a, j}\right)$. Under these conditions the acoustic signal wavelength is given by: $\Lambda_{s}=c_{a} \Delta \tau_{d}=c_{a} / \Delta F_{d}$. iii/ To be in agreement with the PAT-LOFI experimental results shown in the section 4 , the following boundary condition has been used to describe the maximum detected amplitude for the water surface displacement:

$$
A_{a} \frac{Z_{a}}{\min \left(r_{i, j}\right)} \approx 10 \mathrm{~nm}
$$

where $Z_{a}=S_{a} / \Lambda_{s}$ is the acoustic Rayleigh range.

To reconstruct the image $I_{s}(x, y, z)$ of the acoustic sources from the displacement measurements $d_{i}(t)$, the inversion (i.e. the back projection) algorithm which has been used is the one used in [17]:

$$
\begin{gathered}
I_{s}(x, y, z)=\frac{1}{i_{\max }} \sum_{i=1}^{i_{\max }} d_{i}\left[\tilde{\tau}_{i}(x, y, z)\right] W_{i}(x, y, z) \\
W_{i}(x, y, z)=\left[\frac{\tilde{r}_{i}(x, y, z)}{r_{r e f}}\right]^{n}
\end{gathered}
$$

where:

$$
\begin{aligned}
\tilde{\tau}_{i}(x, y, z) & =\frac{\tilde{r}_{i}(x, y, z)}{c_{a}} \\
& =\frac{\sqrt{\left(X_{d, i}-x\right)^{2}+\left(Y_{d, i}-y\right)^{2}+\left(Z_{d, i}-z\right)^{2}}}{c_{a}} \text { is the }
\end{aligned}
$$

time delay for the acoustic propagation between the $i_{\text {th }}$ detection locations $\left(X_{d, i}, Y_{d, i}, Z_{d, i}\right)$ and the spatial position $(x, y, z)$ where the image is reconstructed. In Eq. (3a), the summation is made over the $i_{\max }$ measurement locations. In Eq. (3b), $W_{i}(x, y, z)$ is the dimensionless weighting factor which compensates here the attenuation of the acoustics waves due to the propagation from the acoustics sources to the detection location. $r_{r e f}$ is a normalization distance which can be chosen arbitrary. Indeed this term is simply a multiplicative constant for the whole reconstructed image. In our numerical simulation, for the simplicity of our analytical calculations, we have chosen: $r_{r e f}=Z_{a}$.

The maximum size of the reconstructed images is not only linked to the detection positions, but is given by the condition: $\tilde{\tau}_{i}(x, y, z)<T_{\max }$, where $T_{\max }$ is the duration of the temporal acquisition of the surface displacement $d_{i}(t)$. Also, the possible minimum pixel size is linked to the laser pulse width: $c_{a} \Delta \tau_{L}$.

As explained by Carp et al in [17], the image reconstruction given by Eqs. (3a) and (3b) takes advantages of the direct proportionality between the time of arrival of an acoustics signal at a measurement location and the distance between this location and the point of origin of the signal. As depicted in Figs. 2(a) and 2(b), if a large number of detections contain prominent displacement features corresponding to a certain pixel position, the image reconstruction algorithm assigns high source strength to that pixel. Indeed, on each image of Fig. 2, one can observed 3 bright spots corresponding to the reconstruction of the position of the 3 acoustic sources. 
However, even for pixels that contain no acoustic sources, their corresponding time windows in the individual traces will contain a displacement feature if acoustic sources lying elsewhere in the sample are equidistant from the detection position. Thus, even "empty" pixel will be assigned a small non- zero acoustic source intensity. This "ghost" acoustic source is the reason why "arcing" artifacts are seen in images reconstructed using simple back-projection algorithms. To mitigate this issue we have use the Coherence Filtering (CF) introduced by Liao etal in [18] and also used in [17]:

$$
\begin{gathered}
I_{C F}(x, y, z)=I_{s}(x, y, z) C F(x, y, z) \\
C F(x, y, z)=\frac{\left[\sum_{i=1}^{i_{\max }} d_{i}\left[\tilde{\tau}_{i}(x, y, z)\right] W_{i}(x, y, z)\right]^{2}}{i_{\max } \sum_{i=1}^{i_{\max }}\left[d_{i}\left[\tilde{\tau}_{i}(x, y, z)\right] W_{i}(x, y, z)\right]^{2}} \\
=\frac{i_{\max } I_{s}^{2}(x, y, z)}{\sum_{i=1}^{i_{\max }}\left[d_{i}\left[\tilde{\tau}_{i}(x, y, z)\right] W_{i}(x, y, z)\right]^{2}}
\end{gathered}
$$

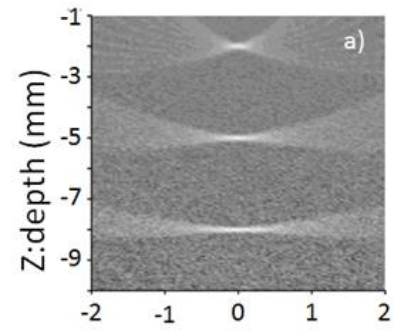

$\mathrm{X}$ :lateral position $(\mathrm{mm})$

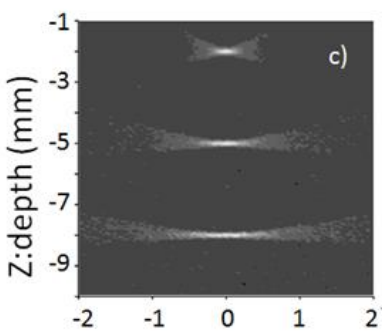

$\mathrm{X}$ :lateral position $(\mathrm{mm})$
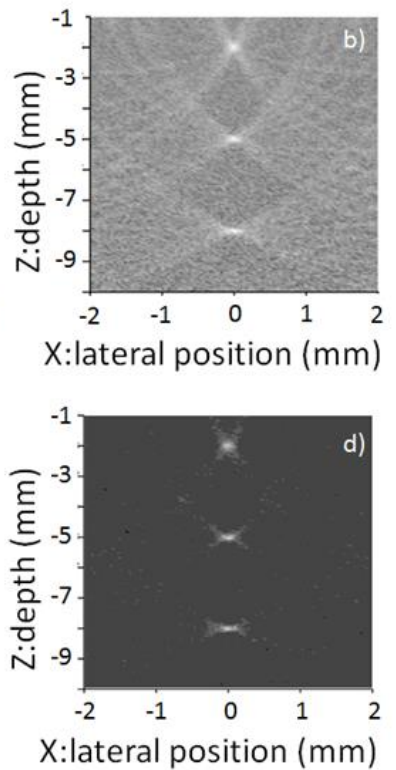

Fig. 2. 2D image reconstructions performed on 3 punctual acoustic sources located on a vertical line below the water surface (i.e. $z=0 \mathrm{~mm}): Z_{s, 1}=-2 \mathrm{~mm}, Z_{s, 2}=-5 \mathrm{~mm}$ and $Z_{s, 3}=-8 \mathrm{~mm}$. The reconstruction is obtained from the surface displacements (induced by the pressure of the acoustic waves) measured at 11 distinct lateral positions $X_{d_{i}}$ along a line segment on the water surface. Left column (a,c): Images reconstructed when $X_{d, i} \in[-2 \mathrm{~mm}, 2 \mathrm{~mm}]$ with an increment of $0.4 \mathrm{~mm}$. Right column $(b, d)$ : Images reconstructed when $X_{d, i} \in[-10 \mathrm{~mm}, 10 \mathrm{~mm}]$ with an increment of $2 \mathrm{~mm}$. Top row $(\mathrm{a}, \mathrm{b})$ : Images reconstructed without the coherence filtering: $I_{S}(x, y=0, z)$. Bottom row (c, d): Images reconstructed with the coherent filtering: $I_{C F}(x, y=0, z)$.
As we can see in Figs. 2(c) and 2(d), the multiplication of $I_{s}(x, y, z)$ by $C F(x, y, z)$ reduces the acoustic source intensity assigned to location $(x, y, z)$ by Eqs. (3a) and (3b), if the location does not contain a real acoustic source. Indeed, if at the location $(x, y, z)$, the reconstructed average intensity corresponds the intersection of $m \leq i_{\max }$ circles, ones obtains:

$$
\begin{aligned}
& I_{s, m}=\frac{m}{i_{\max }} A_{a}, \\
& C F_{m}=\frac{m}{i_{\max }}, \\
& I_{C F, m}=I_{s, m} C F_{m}=\left(\frac{m}{i_{\max }}\right)^{2} A_{a} .
\end{aligned}
$$

Therefore, the ratio between the reconstructed intensities of a real acoustic source $\left(m_{\text {real }}=i_{\max }\right)$ and of a ghost source $\left(m_{\text {ghost }}<i_{\max }\right)$ is increased by taking into account the coherence factor: $\frac{I_{C F, i_{\max }}}{I_{C F, m_{\text {ghost }}}}=\left(\frac{i_{\max }}{m_{\text {ghost }}}\right)^{2}=\frac{i_{\max }}{m_{\text {ghost }}} \frac{I_{s, i_{\max }}}{I_{s, m_{\text {ghost }}}}>\frac{I_{s, i_{\max }}}{I_{s, m_{\text {ghost }}}}$. The final result is a reduction in arcing artifacts and an improvement of the contrast of the reconstructed image.

\section{B. PSF of the reconstructed images}

In Fig. 2, the left and right columns show, for the same number ( $j_{\text {max }}=11$ ) of detection locations, the influence of the size of the detection zone on the dimensions of the PSF (i.e. the area of each reconstructed sources).

As we can see, the PSF area is defined by the intersection of the circles (more precisely the rings of thickness $\Lambda_{s}$ ) which allows reconstructing the punctual sources from the back-projection of the temporal displacement measurements. More precisely, our 2D numerical simulation is made with $j_{\max }=3$ punctual sources aligned vertically below the water surface: $Z_{s, 1}=-2 \mathrm{~mm}$, $Z_{s, 2}=-5 \mathrm{~mm}, \quad Z_{s, 3}=-8 \mathrm{~mm}, \quad$ with $\quad X_{s, j}=0 \mathrm{~mm}$ and $Y_{s, j}=0 \mathrm{~mm}$ for all the sources locations. The detection of the displacement induced by the acoustic waves is made at $i_{\max }=11$ positions along a horizontal $\mathrm{X}$ line:

$-\Delta X_{d} \leq X_{d, i} \leq+\Delta X_{d}, Y_{d, i}=0 \mathrm{~mm}, Z_{d, i}=0 \mathrm{~mm}$,

where $2 \Delta X_{d}=\left|X_{d, i_{\max }=11}-X_{d, 1}\right|$ is the length of the detection segment.

For the reconstructed images shown in the left column [Figs. 2(a) and 2(c)] the detection has been made with $2 \Delta X_{d}=4 \mathrm{~mm}$ while for the right column images [Figs. 2(b) and 2(d)] the detection has been made with $2 \Delta X_{d}=20 \mathrm{~mm}$. 
In our numerical simulation, in order to be in agreement with our experimental conditions (see section 4), the surface displacement $d_{i}(t)$ is calculated for: $0 \mu s \leq t \leq T_{\max }=100 \mu s$. Under this condition, the reconstruction of the image is roughly possible in a disk with a radius of $R=\sqrt{x^{2}+z^{2}} \approx c_{a} T_{\max }=15 \mathrm{~cm}$ centered on the central detection position $\left(X_{d}=0, Z_{d}=0\right)$. Therefore, a square image with a length side of $15 \mathrm{~cm} / \sqrt{2} \approx 10 \mathrm{~cm}$ can be reconstructed. For an easier comparison of the PSF of the different images, we have chosen to show the reconstruction images in windows with the same size, whatever the detection segment size.

From the size of the detection segment, we define empirically the "mean" synthetic numerical aperture, for a given source depth $\left(Z_{s}\right)$ by:

$$
N A\left(Z_{s}\right) \approx \frac{\Delta X_{d} / 2}{\sqrt{\left(\Delta X_{d} / 2\right)^{2}+Z_{s}^{2}}}
$$

By using Eq. (7), the lateral and in-depth PSFs $\left(\delta_{X}, \delta_{Z}\right)$ of the reconstructed source can be defined and are respectively given by:

$$
\begin{aligned}
& \delta_{X}\left(Z_{s}\right) \approx \frac{\Lambda_{s}}{N A\left(Z_{s}\right)} \\
& \delta_{Z}\left(Z_{s}\right) \approx \frac{\Lambda_{s}}{\sqrt{1-N A^{2}\left(Z_{s}\right)}}
\end{aligned}
$$

where $\Lambda_{s}=c_{a} \Delta \tau_{d}=150 \mu \mathrm{m}$ the characteristic wavelength of the detected acoustic pulse (which corresponds to a detection bandwidth $\Delta F_{d}=10 \mathrm{MHz}$ ). Finally, by using Eqs. (8a) and (8b) the analytic expression of the reconstructed image obtained from Eq. (3a) is equal to:

$$
\begin{aligned}
& I_{s}(x, y=0, z) \approx \\
& \sum_{j=1}^{j_{\max }} \exp \left(-2\left[\left(\frac{x}{\delta_{X}(z)}\right)^{2}+\left(\frac{z-Z_{s, j}}{\delta_{Z}(z)}\right)^{2}\right]\right)
\end{aligned}
$$

where the Gaussian shape of the reconstructed sources come from the Gaussian shape of the of acoustic signal temporal profile [see Eq. (1)]

In agreement with Eqs. (7), (8a) and (8b), one can observe on Fig. 2 how the lateral width of the PSF $\left(\delta_{X}\right)$ is smaller when the numerical aperture ( $N A$ ) increases (i.e. when $\left|\Delta X_{d}\right| \nearrow$ increases or when $\left|Z_{s}\right| \searrow$ decreases), while an opposite evolution occurs for the indepth width of the PSF $\left(\delta_{Z}\right)$. Indeed, $\delta_{Z}$ is larger, when the detection zone is larger (i.e. when $\left|\Delta X_{d}\right| \nearrow$ increases) or when the depth is smaller $\left(\left|Z_{s}\right| \searrow\right.$ decreases).

The best compromise between the lateral and in-depth width is obtain for $N A=\frac{1}{\sqrt{2}}$ (i.e. for: $\Delta X_{d} / 2=Z_{s}$ ) and under this condition, one obtains:

$$
\delta_{X}=\delta_{Z}=\sqrt{2} \Lambda_{s}=212 \mu m
$$

\section{Signal to Noise Ratio (SNR) of the reconstructed image}

The image reconstruction shown in Fig. 2 has been obtained from a simulated signal with an additive noise. Indeed, for each displacement $d_{i}(t)$ a noise $\Delta d_{i}(t)$ has been added with an average and a standard deviation which are respectively given by:

$$
\begin{aligned}
& \left\langle\Delta d_{i}(t)\right\rangle=0 \mathrm{~nm} \\
& \sqrt{\left\langle\Delta d_{i}(t)^{2}\right\rangle}=\Delta d=1 \mathrm{~nm}
\end{aligned}
$$

In agreement with the measurements made with our LOFI setup, the noise is therefore independent of the detection position (i.e. independent of the indexi) and its amplitude is in the nanometer range for a single shot measurement (see section 4).

For a given detection position $\left(X_{d}, Y_{d}=0, Z_{d}=0\right)$, the signal to noise ratio $\left(S N R_{d}\right)$ of the interface displacement measurements is defined by the ratio between the maximum value of the time evolution of the measured displacement and the displacement ground noise:

$\operatorname{SNR}_{d}\left(X_{d}, Z_{s}\right)=\frac{\max [d(t)]}{\Delta d}=\frac{A_{a} \frac{Z_{a}}{\sqrt{X_{d}^{2}+Z_{s}^{2}}}}{\Delta d}$

Due to the attenuation of the acoustics waves with the propagation distance the SNR given by Eq. (12) is inversely proportional to the distance between the source location $\left(X_{s}=0, Y_{s}=0, Z_{s}\right)$ and the detection location.

Under our modeling conditions, the maximum and minimum values of $S N R_{d}$ are respectively given by:

$$
\begin{gathered}
\max \left(S N R_{d}\right)=S N R_{d}\left(0, Z_{s, 1}\right)=10 \\
\min \left(S N R_{d}\right)=S N R_{d}\left( \pm \Delta X_{d}= \pm 10 \mathrm{~mm}, Z_{s, 3}\right) \approx 1.6 .
\end{gathered}
$$

Eq. (13a) corresponds to the maximum value of $S N R_{d}$ obtained when the detection distance is minimum. While Eq. (13b), corresponds to the minimum value of $S N R_{d}$ obtained when the detection distance is maximum.

More generally, for a given source depth $\left(Z_{s}\right)$, an intermediate value of $S N R_{d}$ is obtain for an intermediate detection distance, which corresponds to the detection position localized between the center and one of the edges of the detection segment in our numerical simulation:

$$
\begin{aligned}
& S N R_{d, \text { int }}\left(Z_{s}\right)=S N R_{d}\left(X_{d}= \pm \frac{\Delta X_{d}}{2}, Z_{s}\right) \\
& \approx 10 \times \frac{\left|Z_{s, 1}\right|}{\sqrt{Z_{s}^{2}+\left(\Delta X_{d} / 2\right)^{2}}}
\end{aligned}
$$


For the situation where the image will be restored with the best compromise between the lateral and in-depth resolutions (i.e. when $\left|Z_{s, 2}\right|=\Delta X_{d} / 2=10 \mathrm{~mm} / 2$ ), one obtains:

$\operatorname{SNR}_{d, \text { int }}\left(Z_{S, 2}=-\Delta X_{d} / 2\right)=\frac{10}{\sqrt{2}} \times \frac{\left|Z_{s, 1}\right|}{\left|Z_{s, 2}\right|} \approx 3$.

In the rest of this section, our goal is to link the in-depth value of the SNR of the reconstructed image $S N R_{I_{C F}}\left(Z_{s}\right)$ to the intermediate value of signal to noise ratio of the displacement measurement (i.e. $\operatorname{SNR}_{d, \text { int }}\left(Z_{s}\right)$ ).

For the reconstructed source subscripted $\mathrm{j}$, the signal and the variance obtain from Eqs. (3a) and (3b) are respectively given :

$$
\begin{aligned}
& I_{s, j}=I_{s}\left(X_{s, j}, Y_{s, j}, Z_{s, j}\right)=\frac{1}{i_{\max }} \sum_{i=1}^{i_{\max }} A_{a} \frac{Z_{a}}{r_{i, j}} \frac{r_{i, j}}{Z_{a}}=A_{a} \\
& \Delta I_{s, j}^{2}=\frac{1}{i_{\max }^{2}} \sum_{i=1}^{i_{\max }} \Delta d^{2} \frac{r_{i, j}^{2}}{Z_{a}^{2}} \approx \frac{\Delta d^{2}}{i_{\max }}\left[\frac{\left(\Delta X_{d} / 2\right)^{2}+Z_{s, j}^{2}}{Z_{a}^{2}}\right]
\end{aligned}
$$

where for simplicity, we have approximate all the distances $r_{i, j}$ by the intermediate distance $r_{i, j} \approx \sqrt{\left(\Delta X_{d} / 2\right)^{2}+Z_{s, j}^{2}}$.

Finally the SNR of one reconstructed source is given by

$$
\begin{aligned}
\operatorname{SNR}_{I_{s}}\left(Z_{S}\right) & =\frac{I_{s}}{\Delta I_{s}\left(Z_{s}\right)} \approx \frac{A_{a} Z_{a}}{\sqrt{\left(\Delta X_{d} / 2\right)^{2}+Z_{s}^{2}}} \frac{\sqrt{i_{\max }}}{\Delta d} \\
& =\sqrt{i_{\max }} S N R_{d, \text { int }}\left(Z_{s}\right)
\end{aligned}
$$

The SNR increase, given by $\sqrt{i_{\max }}$, comes from the average of displacement measurements with white noise.

If the coherence factor is taken into account, one finally obtains (see Eqs (4a), (4b) and (5) with $m_{\text {gost }}=i_{\max }$ for the signal and $m_{\text {gost }}=1$ for the noise):

$$
\begin{aligned}
& I_{C F}=I_{s} \\
& \Delta I_{C F} \approx \frac{3}{i_{\max }} \Delta I_{s}
\end{aligned}
$$

and finally:

$$
\operatorname{SNR}_{I_{C F}}\left(Z_{s}\right)=\frac{I_{C F}}{\Delta I_{C F}\left(Z_{s}\right)} \approx \frac{i_{\max }^{3 / 2}}{3} \operatorname{SNR} R_{d, \text { int }}\left(Z_{s}\right)
$$

Even if the calculation details are not shown here, Eq. (18b) is an approximate formula which can be obtain from a noise propagation calculation. Indeed, starting from the displacement noise $(\Delta d)$, one can obtained after straightforward but tedious derivative calculations the noises $\Delta I_{s}$ (see Eq. (16b) and $\Delta I_{C F}$ according to $\Delta d$, and finally
Eq. (18b) by keeping only the dominant contribution. The validity of this equation has been checked numerically for different numerical conditions corresponding to potential experimental conditions.

Roughly speaking, in Eq. (18b) (and consequently in Eq. (19)), the multiplicative factor 3 and comes from the fact that $I_{C F} \propto I_{s}^{3}$ [see Eqs. (4a) and (4b)], and the division by $i_{\text {max }}$ comes from the coherence factor which induces for the random noise, the same attenuation than the one for the arcing effect with: $m_{\text {gost }}=1$.

In the following of this manuscript, the goal is to check the analytical expressions of the widths [given by Eqs. (8a) and (8b)] and of the signal to noise ratio [given by Eq. (19)], of the reconstructed acoustic sources, firstly obtained with numerical simulations (Figs. 2 and 3) and secondly obtained with our experimental results (Fig. 4).

Fig. 3(a) shows 3 surface displacements (among the 11) induced by the acoustic waves emitted by the 3 punctual sources. For each displacement simulated, one can observe the arrival times of the acoustic impulsions emitted by the 3 sources located at different depths: $Z_{s, 1}=-2 \mathrm{~mm}, Z_{s, 2}=-5 \mathrm{~mm}$ and $Z_{s, 3}=-8 \mathrm{~mm}$ below the sample surface (i.e. $z=0 \mathrm{~mm}$ ). Due to the acoustic wave attenuation with the propagation distance, the displacement amplitude decreases with the arrival time $\tau_{i, j}=r_{i, j} / c_{a}$. In agreement with Eqs. (13a) and (13b) the signal to noise ratio of the first displacement pulse is maximum and equals to $\max \left(S N R_{d}\right)=10 \pm 1$ while it is minimum $\min \left(S N R_{d}\right) \approx 2 \pm 1$ for the latest one. While for the second peak of the middle trace the SNR is roughly equal to: $\operatorname{SNR}_{d, \text { int }}\left(Z_{s, 2}\right) \approx 3 \pm 1$.

Figs. 3(b) and 3(c), show the in-depth reconstruction of the acoustic sources positions obtained respectively without the coherence filtering $\left(I_{s}(x=0, z)\right)$ and with the coherence filtering $I_{C F}(x=0, z)$. One can notice that Figs. 3(b) and 3(c) correspond respectively to the central vertical line of the images of Figs. 2(b) and 2(d). In agreement with Eq. (8b), the two figures clearly show that that the in-depth width of the reconstructed image $\left(\delta_{Z}(Z)\right)$ decreases with the depth. We further observed that the weighting factor allows to restore the amplitudes of the wave emitted by the acoustic sources (which are the same in our numerical simulation: $A_{S, j}=A_{a}$ ), counterbalanced by an increase of the in-depth noise.

Regarding the noise, the comparison of Figs. 3(b) and 3(c) clearly shows that the coherence factor reduces the noise level and therefore increases the contrast of the reconstructed sources. In agreement with Eqs. (17) and (19), the signal to noise ratio of the reconstructed central acoustic source is: $\quad S N R_{I_{s}} \approx 3 \sqrt{11} \approx 10 \quad$ and $S N R_{I_{C F}} \approx 3 \frac{11^{3 / 2}}{3} \approx 36$. 

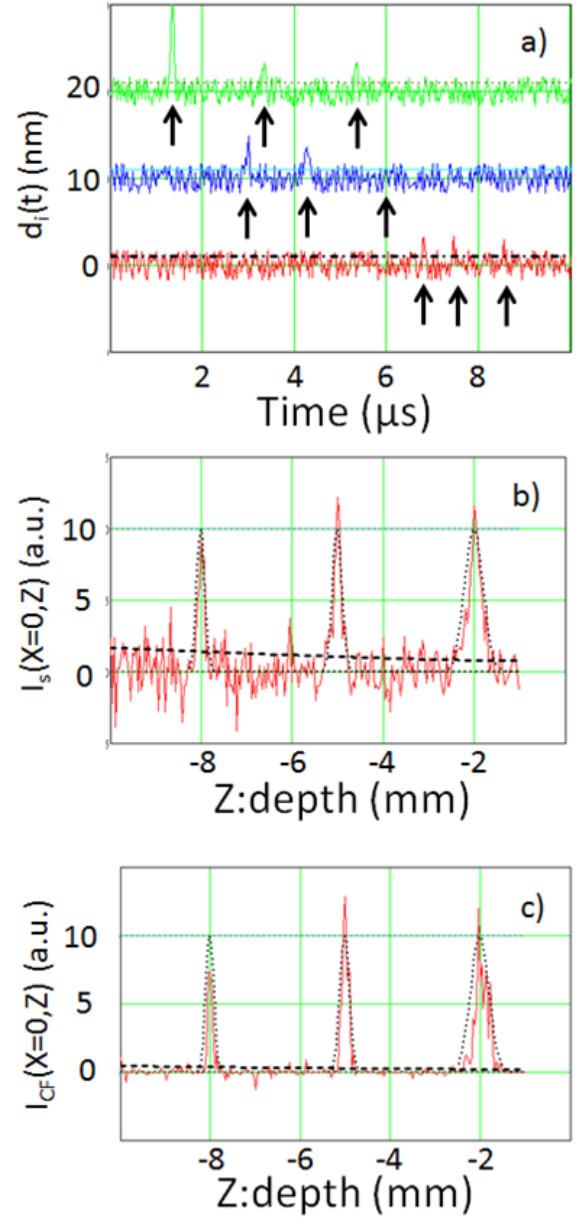

Fig. 3. a) Displacement measurements $d_{i}(t)$ induced by the 3 punctual acoustic sources located at: $Z_{s, 1}=-2 \mathrm{~mm}, Z_{s, 2}=-5 \mathrm{~mm}$ and $Z_{s, 3}=-8 \mathrm{~mm}$ below the water surface with $X_{s, j}=0 \mathrm{~mm}$. Lower trace: $d_{1}(t)$ measured at $X_{d, 1}=-\Delta X_{d}=-10 \mathrm{~mm}$; Middle trace: $d_{4}(t)$ measured at $X_{d, 4}=-4 \mathrm{~mm}$; Upper trace: $d_{6}(t)$ measured at $X_{d, 6}=0 \mathrm{~mm}$. For clarity the different measurements are vertically shifted by a 10 nanometer step. For each displacement measurement, the 3 black arrows indicate the arrival times of the acoustic impulsions emitted by the 3 sources and the horizontal dashed-line is the displacement noise level $\Delta d=1 \mathrm{~nm}$. b) In depth reconstruction of the acoustic sources positions obtained without the coherence filtering: $I_{S}(x=0, z)$. The dashed line is the noise level given by Eq. (16b).The dotted line is the in-depth reconstructed signal given by the analytical expression of Eq. (9). c) In depth reconstruction of the acoustic source position obtained with the coherence filtering: $I_{C F}(x=0, z)$. The dashed line is the noise level obtained from Eqs. (18b) and (16b). The dotted line is the in-depth reconstructed signal by Eq. (9).

N.B. The in depth reconstruction shown in b) and c) are obtained from the whole 11 surface displacement measurements and not only from the 3 displacements shown in a).

\section{IN-DEPTH IMAGE RECONSTRUCTION PERFORMED FROM THE LOFI SIGNAL}

\section{A. Experimental conditions}

With a LOFI setup (Fig.1), we have measured the transient displacements of the air/water interface which are induced by the pressure waves generated by the photoacoustic (PA) effect.

To generate, the PA waves, the green light of a pulsed Nd:YAG laser with an intra-cavity frequency doubling (i.e. $\lambda_{P A}=532 \mathrm{~nm}$ ) has been sent on an absorbing phantoms composed of two parallel polyamide tubes filed with red ink, which roughly mimic large blood vessels. The two parallel tubes with an internal and an external diameters respectively equal to: $D_{\text {int }}=800 \mu \mathrm{m}=D_{a}$ and $D_{\text {ext }} \approx 1 \mathrm{~mm}$, are separated by a distance (center to center) of $D_{c c} \approx 2.5 \mathrm{~mm}$ ) and are parallel to the water surface $(X, Y)$ plane. The centroid of the two tubes is submerged at a depth of $Z_{c} \approx-3.5 \mathrm{~mm}$ under the water surface. Fig. 1 shows that the axes of the tubes which are parallel to the $Y$ direction, are perpendicular to the green PA laser illumination which is parallel to the $\mathrm{X}$ direction. The plane defined by the axes of the two parallel tubes is tilted by an angle of $\alpha \approx 15^{\circ}$ relative to the water surface. This tilt allows the simultaneous optical illumination of the two absorbing tubes with the green laser light (see Fig. 1).

The green laser used to generated the PA absorption has a pulse energy of $Q_{P A}=2 \mathrm{~mJ}$ (controlled by a half wave plate and a polarizer), a pulse width of $\Delta \tau_{L}=10 \mathrm{~ns}$, a repetition rate of $10 \mathrm{~Hz}$ and consequently an average power of $P_{P A}=20 \mathrm{~mW}$. The laser beam section area has been extended to $S_{P A}=20 \mathrm{~mm}^{2}$ (i.e. the diameter is $D_{P A}=5 \mathrm{~mm}$ ) in order to allow the simultaneous illumination of the two polyamide tubes with a surface energy density which is compatible with the maximum permissible exposure (MPE) defined by the American National Standard Institute (ANSI) safety standard for future biomedical measurements [19]:

$$
H_{P A}=10 \mathrm{~mJ} / \mathrm{cm}^{2} \leq M P E=20 \mathrm{~mJ} / \mathrm{cm}^{2}
$$

For the LOFI detection of the transient displacement of the air/water interface, we have used a CW Nd: YAG ( $\lambda_{L}=1064 \mathrm{~nm}$ ) laser, with an output power of $P_{\text {out }}=20 \mathrm{~mW}$ and a relaxation oscillation frequency of $F_{R}=1.1 \mathrm{MHz}$. The frequency shift of the optical feedback is tuned to $F_{e}=5 \mathrm{MHz}$, in order to be able to record at the shot noise level a vibration spectrum of several $\mathrm{MHz}$ [14]. Experimentally the feedback level can be controlled by playing with the efficiency of the frequency shifter (composed of two acousto-optic deflectors) and allows to work in the weak feedback regime where the LOFI vibrometer performs linearly [13-14]. The laser output power modulation induced by the frequency shifted optical feedback is detected by using a reversed bias PIN photodiode (THORLABS/DET110) and the delivered voltage is send to a DAQ card (SPECTRUM/M3I.4111) and processed by a PC to finally obtain the displacement measurements $\left(d_{i}(t)=d_{a}\left(X_{d, i}, Y_{d, i}, t\right)\right)$ [14]. For each detection location $\left(X_{d, i}, Y_{d, i}, Z_{d, i}=0\right)$ on the water surface, the total acquisition time is $T_{a c q}=81.92 \mu \mathrm{s}$ at a sampling rate of $100 \mathrm{MHz}$, which is compatible with the laser pulse width 
$\Delta \tau_{L}=10 \mathrm{~ns}$. The temporal evolution of the surface displacement is filtered with a band-pass filter with a half width $\Delta F_{d}$ chosen to be compatible with the time-width of the acoustic pulses emitted by the polyamide tubes: $\Delta F_{d}=1.3 \mathrm{MHz} \approx \frac{2}{\pi} \frac{c_{a}}{D_{a}}=1.2 \mathrm{MHz}$ [14].

At this point, one can notice that the sampling rate is very high by comparison with the detection bandwidth used to image the polyamide tubes. This sampling rate has been chosen to be able in future experiments to detect smaller objects by using a higher band pass filter.

Experimentally, the LOFI laser beam which is focused on the water surface has a power $P_{L} \approx 1 \mathrm{~mW}$ (controlled by the efficiency of the frequency shifter) and a radius of $r_{L} \approx 20 \mu \mathrm{m}$. Under these experimental conditions, one can notice that the surface energy density of our LOFI focalized beam is is 76 times lower than the MPE limit. For biomedical applications [19]:

$$
\begin{aligned}
H_{L}\left(T_{a c q}\right) & =\frac{P_{L} \times T_{a c q}}{\pi r_{L}^{2}}=\frac{7 \mathrm{~mJ}}{\mathrm{~cm}^{2}} \\
& <<\operatorname{MPE}\left(T_{a c q}\right)=5500 \times T_{a c q}^{0.25}=\frac{530 \mathrm{~mJ}}{\mathrm{~cm}^{2}}
\end{aligned}
$$

\section{B. Analysis of the reconstructed experimental image}

Fig. 4 shows the experimental PAT images of the two polyamide tubes obtained from the inversion the LOFI displacement measurements. Due to the translational invariance of the polyamide tube along its axis which is oriented in the Y direction (see Fig.1), the tomographic image shown here is simply a 2D in-depth image $(x, z)$ reconstructed from a 1D LOFI temporal acquisitions along the horizontal $x$ line. The pixel size of the reconstructed images is fixed to: $15 \mu m \times 15 \mu m$, in agreement with both the laser pulse width and the sampling rate of the DAQ card $: \Delta \tau_{L}=10 \mathrm{~ns}=1 / 100 \mathrm{MHz}$ leading to: $c_{a} \Delta \tau_{L}=15 \mu m<<D_{a}$.

Fig. 4(a) shows the temporal displacements of the air/water interface ( $d_{i, \exp }(t)$ ) measured with our LOFI setup focused at 20 different positions on the water surface. One can visualize the two curved wavefronts corresponding to propagation time of the pulsed-acoustic waves emitted by the two polyamide tubes. For each wave-front, one can also observe how the displacement amplitudes decrease with the arrival times (i.e. with the propagation distance between the detection position and the acoustic sources). Experimentally, an attenuation proportional to: $1 / r^{n}$ with $n \approx 1.6 \pm 0.2$ has been determined. One can notice that this attenuation is stronger than the one of cylindrical wave ( $n=0.5$ ) which is the kind of wave normally emitted by the polyamide tube. This can be explained by the fact that when the detection position is not just above the acoustic sources, the pressure wave front is not parallel to the water surface and consequently the vertical water surface displacement is reduced by a cosine effect [17].
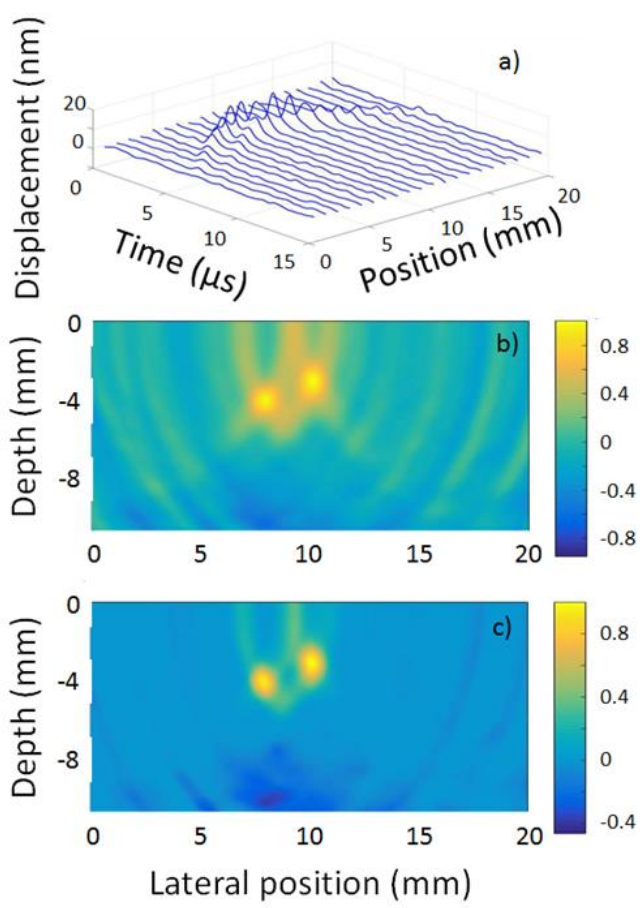

Fig. 4: PAT based on LOFI performed on a phantom composed of two polyamides tubes with an internal diameter of $800 \mu \mathrm{m}$, separated by a distance of $2.5 \mathrm{~mm}$ and submerged at a mean distance of $-3.5 \mathrm{~mm}$ depth under the water surface. a) Temporal displacement $d_{i}(t)$ of one point of the water surface measured at 20 different detection positions along a line perpendicular to the tube axes. Each measurement is in an average of nine acquisitions with an average displacement noise of $0.35 \mathrm{~nm}$. b) Normalized reconstructed image $\left(\frac{I_{S}(x, y=0, z)}{\max \left[\left[I_{S}(x, y=0, z)\right]\right]}\right)$, based on Eqs. (3a) and (3b) with $n=1.6$ and $r_{r e f}=3 \mathrm{~mm}$. c) Normalized reconstructed image $\left(\frac{I_{C F}(x, y=0, z)}{\max \left[\left[I_{C F}(x, y=0, z)\right]\right]}\right)$ based on Eqs. (4a) and (4b).

In Fig. 4(a), a more detail study of the displacement signal measured at the detection position: $X_{d}=10 \mathrm{~mm}$ (which is the nearest to the polyamides tubes), shows principally two acoustic pulses with arrival times of: $\tau_{10,1}=1.95 \mu \mathrm{s}$ and $\tau_{10,2}=3.17 \mu \mathrm{s}$, and with temporal widths of: $\Delta \tau_{10,1}=0.54 \mu \mathrm{s}$ and $\Delta \tau_{10,2}=0.47 \mu \mathrm{s}$. The two arrival times don't allow to determine the spatial positions of the two polyamide tubes but simply indicate that the radial distance between the central detection position and the two acoustic sources are respectively given by: $r_{10,1}=c_{a} \tau_{10,1}=2.96 \mathrm{~mm} \quad$ and $r_{10,2}=c_{a} \tau_{10,2}=4.81 \mathrm{~mm}$ with $c_{a}=1520 \mathrm{~ms}^{-1}$. On the other hand, due to the experimental condition $\left(\Delta \tau_{L}<<D_{a} / c_{a}\right)$ and a welladapted bandpass filter, the temporal widths of the acoustic pulses 
allow to estimate the diameters of the two polyamides tubes (i.e. of the two acoustic sources):

$$
\begin{aligned}
& D_{s, 1}=c_{a} \Delta \tau_{10,1}=820 \mu \mathrm{m} \pm 15 \mu \mathrm{m}, \\
& D_{s, 2}=c_{a} \Delta \tau_{10,2}=714 \mu \mathrm{m} \pm 15 \mu \mathrm{m} .
\end{aligned}
$$

One can observed that the second one is a little bit smaller than the first one, probably due to shading effect of the PA optical illumination. One can also notice that the detected widths of the polyamide tubes are in good agreement with the real one $\left(D_{s, 1}, D_{s, 2} \approx D_{a}=800 \mu \mathrm{m}\right)$.

In Fig. 4(a), each displacement trace is an average of $N=9$ measurements made at the same detection position and with a resulting noise of $\Delta d_{\text {exp }} \approx 0.35 \mathrm{~nm} \quad(\approx 1 \mathrm{~nm}$ for a single shot measurement). This noise level is in a very good agreement with the theoretical prediction for a LOFI setup [14]:

$$
d_{a, \min }(N=9)=\frac{1}{\sqrt{N}} \frac{\lambda_{L}}{2 \pi} \frac{\sqrt{2 \Delta F_{e}}}{\sqrt{R_{e} P_{\text {out }} / \frac{h c}{\lambda_{L}}}} \approx 0.28 \mathrm{~nm}
$$

for a filtering bandwidth of $\Delta F_{e}=1.3 \mathrm{MHz}$ and a feedback effective power reflectivity of: $R_{e} \approx 10^{-6}$, experimentally determined from the study of the LOFI SNR [14-16].

The corresponding overpressure induces by the acoustic wave is therefore equal to:

$$
\begin{aligned}
p_{a, \min }(N=1) & =\frac{Z_{\text {eau }} d_{a, \min }(N=1)}{D_{a} / c_{a}} \\
& \approx Z_{\text {eau }} d_{a, \min }(N=1) \Delta F_{d} \approx 3 \mathrm{kPa}
\end{aligned}
$$

where $Z_{\text {eau }}=1.5 \times 10^{6} \mathrm{~Pa} \mathrm{~s} \mathrm{~m}^{-1}$ is the acoustic impedance of water. At this point, one can noticed that the value of $p_{a \text {, min }}$ given by Eq. (23b) and obtain from the LOFI measurement is at least two order of magnitude higher than the value of the noise equivalent pressure ( $N E P \approx 10 P a$ ) which can be detected by using a PZT detector with an equivalent detection bandwidth [20-21]. The LOFI interferometer which has the advantages to allow non-contact measurement of the surface displacement has the drawback to be less sensitive than the widely used PZT for the detection of acoustic waves.

Nevertheless, Eq. (23a) shows that the sensitivity of our LOFI setup can be improved by increasing the laser output power. If the power of the LOFI laser is increased by a multiplicative factor equals to 76 to reach the MPE limit (see Eq. 21) than the detection bandwidth can be increased by the same factor $\left(76 \times \Delta F_{d} \approx 100 \mathrm{MHz}\right)$ to obtain the same sensitivity for the detection of the displacement amplitude (i.e. 1 $\mathrm{nm}$ for a single shot measurement). To be able to use such bandwidth, the carrier frequency of our LOFI setup needs to be shifted to: $F_{e}=80 \mathrm{MHz}$, by using a unique acousto-optic modulator.

Also, if we keep the same detection bandwidth, the noise of the displacement amplitude decreases to: $1 \mathrm{~nm} / \sqrt{76} \approx 0.11 \mathrm{~nm}$ if the laser power is increased $(\times 76)$.
Eq. (23a) also shows that the sensitivity of our LOFI setup can be improved by the average of $N$ displacement measurements. Nevertheless, with the increase of the exposure time to: $N \times T_{a c q}$, the focalized LOFI energy density need to stay below the MPE value. For example, for an average of $N=N_{\text {max }}=350$ measurements, the limit of the MPE condition is reached if the laser is kept to: $P_{L} \approx 1 \mathrm{~mW}$

$$
\begin{aligned}
H_{L}\left(N \times T_{a c q}\right) & =\frac{P_{L} \times N \times T_{a c q}}{\pi r_{L}^{2}}=\frac{2.3 \mathrm{~J}}{\mathrm{~cm}^{2}} \\
& =M P E=5500 \times\left(N \times T_{a c q}\right)^{0.25}=\frac{2.3 \mathrm{~J}}{\mathrm{~cm}^{2}}
\end{aligned}
$$

Under this condition the minimum measurable displacement is given by:

$$
d_{a, \min }(N=350) \approx 5 \times 10^{-11} \mathrm{~m},
$$

which corresponds to the following overpressure induces by the acoustic wave:

$$
p_{a, \min }\left(N_{\max }=350\right) \approx 3 \mathrm{kPa} / 20=150 \mathrm{~Pa}
$$

Therefore, the sensitivity of the LOFI sensor can becomes better, but at the expense of the signal acquisition time and therefore a decrease of the imaging frame-rate.

From the signal measured at the detection position which the nearest to the polyamides tubes $\left(X_{d}=10 \mathrm{~mm}\right.$ ), one detect the highest displacement peak which is roughly equal to: $\approx 12 \mathrm{~nm}$ and therefore the maximum value of $S N R_{d}$ is:

$$
\max \left(S N R_{d, \exp }\right)=12 \mathrm{~nm} / 0.35 \mathrm{~nm} \approx 34
$$

At the intermediate detection position ( $X_{d}=5 \mathrm{~mm}$ ), one observe a displacement peak roughly equals to: $\approx 3.5 \mathrm{~nm}$ and therefore the intermediate value of $S N R_{d}$ is equal to:

$$
S N R_{d, \text { int,exp }} \approx 3.5 \mathrm{~nm} / 0.35 \mathrm{~nm}=10
$$

In Fig. 4(a), the 20 displacement traces have been measured along the horizontal X line (see Fig. 1) with a position increment of $1 \mathrm{~mm}$, but to obtain the reconstructed images shown in Figs. 4(b) and (c), only the 18 displacement measurements (among the 20 realized) with the best value of $S N R_{d_{i}}$ has been used. The detection segment length is therefore equals to:

$$
2 \times \Delta X_{d, \exp }=(18-1) \times 1 \mathrm{~mm}=17 \mathrm{~mm}
$$

So, the acoustic waves emitted by the two polyamide tubes immerged at a central depth of: $Z_{c}=-3.5 \mathrm{~mm}$, are detected with a numerical aperture given by [see Eq. (7)]:

$$
N A\left(Z_{c}=-3.5 \mathrm{~mm}\right) \approx \frac{\Delta X_{d, \exp } / 2}{\sqrt{\left(\Delta X_{d, \exp } / 2\right)^{2}+Z_{c}^{2}}}=0.77
$$

Fig. 4(b) shows the in-depth image reconstruction performed by using Eq. (3a) with the LOFI displacement measurements $\left(d_{i, \exp }(t)\right)$ and 
with the dimensionless weighting factor given by Eq. (3b) with $n=1.6$ and $r_{r e f}=\min \left(r_{i, j}\right)=r_{10,1} \approx 3 \mathrm{~mm}$. Respectively, Fig. 4(c) shows the in-depth image reconstruction performed by using Eqs. (4a) and (4b).

In Figs. 4(b) and 4(c), the 2D reconstructions (X,Z) clearly show the image of the section of two polyamide tubes. The comparison of Figs. 4(b) and 4(c) clearly show how the use of the coherence factor allows to reduce the arcing noise. By comparison with our numerical simulation, one can observed some arcing residues in the final experimental image. This residue can be explained by the fact that the experimental images are reconstructed from displacement measurements with higher values $S N R_{D_{i}}$ than those of the numerical simulation. Consequently, for the numerical simulation, the arcing residues are below the background noise level while for the experimental measurements they remain higher than the background noise.

In very good agreement with our experimental configuration, Fig. 4(c) shows, that, the reconstructed coordinates of the polyamide tubes centers are:

$$
\begin{aligned}
& X_{s, 1}=9.86 \mathrm{~mm} \pm 15 \mu \mathrm{m} ; Z_{s, 1}=-3.08 \mathrm{~mm} \pm 15 \mu \mathrm{m} \\
& X_{s, 2}=12.32 \mathrm{~mm} \pm 15 \mu \mathrm{m} ; Z_{s, 2}=-3.93 \mathrm{~mm} \pm 15 \mu \mathrm{m}
\end{aligned}
$$

Consequently, the distance (center to center) and the coordinates of the centroid of the two polyamide tubes are respectively given by:

$$
\begin{gathered}
D_{c c}=2.5 \mathrm{~mm} \pm 20 \mu \mathrm{m} \\
X_{c}=11.09 \mathrm{~mm} \pm 20 \mu \mathrm{m} ; Z_{c}=-3.50 \mathrm{~mm} \pm 20 \mu \mathrm{m} .
\end{gathered}
$$

For the two polyamide tubes, the lateral $\left(D_{X}\right)$ and the in-depth $\left(D_{Z}\right)$ experimental widths of the reconstructed tube section are respectively equal to:

$$
\begin{aligned}
& D_{X, 1}=1080 \mu \mathrm{m} \pm 19 \mu \mathrm{m} ; D_{X, 2}=1050 \mu \mathrm{m} \pm 21 \mu \mathrm{m} \\
& D_{Z, 1}=1500 \mu \mathrm{m} \pm 25 \mu \mathrm{m} ; D_{Z, 2}=1260 \mu \mathrm{m} \pm 22 \mu \mathrm{m}
\end{aligned}
$$

These values are in relatively good agreement with the theoretical prediction given by: Eqs (8a) and (8b):

$$
\begin{aligned}
& D_{X, 1}=\frac{D_{s, 1}}{N A\left(Z_{s, 1}\right)}=1035 \mu \mathrm{m} \pm 19 \mu \mathrm{m}, \\
& D_{X, 2}=\frac{D_{s, 2}}{N A\left(Z_{s, 2}\right)}=1001 \mu \mathrm{m} \pm 21 \mu \mathrm{m} ; \\
& D_{X, 1}=\frac{D_{s, 1}}{\sqrt{1-N A^{2}\left(Z_{s, 1}\right)}}=1344 \mu \mathrm{m} \pm 25 \mu \mathrm{m}, \\
& D_{Z, 2}=\frac{D_{s, 2}}{\sqrt{1-N A^{2}\left(Z_{s, 2}\right)}}=1019 \mu \mathrm{m} \pm 22 \mu \mathrm{m},
\end{aligned}
$$

where $D_{s, 1}$ and $D_{s, 2}$ are given by Eq. (22).
From Fig. 4(c), one could also determine the signal to noise ratio (SNR) of the reconstructed image. For each tube, the SNR is simply given by the ratio between the maximum signal value and the noise level obtained by the calculation of the standard deviation of an horizontal line of 600 pixels located at the depth $Z_{s, i}$, but outside the reconstructed zone of the polyamide tube:

In agreement with the theoretical predictions given by Eqs. (17) and (19), one can obverse that the SNR decreases with the reconstruction depth:

$S N R_{I_{C F}, \exp }\left(Z_{s, 1}\right) \approx 243 ; S N R_{I_{C F}, \exp }\left(Z_{s, 2}\right) \approx 193$,

Also, these results are roughly in good agreement with the calculated value obtained by combining Eq. (26) with Eq. (19):

$S N R_{I_{C F}}\left[\left(Z_{s, 1}+Z_{s, 2}\right) / 2\right] \approx \frac{i_{\max }^{3 / 2}}{3} S N R_{d, \text { int,exp }} \approx 254$,

with $i_{\max }=18$.

Here, let us recall that that the image of Figs. 4(b) and 4(c) has been reconstructed by keeping only the 18 displacement measurements (among the 20 realized measurements shown in Fig. 4(a) with the best value of $S N R_{d_{i}}$. If the two displacement measurements obtained from detection positions located farthest from the polyamide tubes (i.e. with the lowest $S N R_{d_{i}}$ values) are added for the image reconstruction, one obtained a reconstructed image with roughly the same width for the section of the polyamide tubes (i.e. due to the fact that the number of measurements which principally contribute to the reconstruction signal remains constant) but with a lower $S N R_{I_{C F}}$ (induced by an additive increase of noise). So, the number of measures used in the reconstruction process is an important parameter to optimize the quality of the reconstructed acoustic sources (SNR and width).

\section{CONCLUSION AND PERSPECTIVES}

In this paper we have demonstrated that a Laser Optical Feedback Imaging (LOFI) setup can be used for the optical detection of ultrasound in Photo-Acoustic Tomography (PAT).

For PAT, the images are reconstructed from the optical measurements of surface displacements made at several locations just after the optical irradiation of the absorbing sample with a pulsed laser. We show that the LOFI vibrometer based on a CW microchip Nd:YAG laser with a relaxation frequency in the megahertz range, provides surface displacement measurements a displacement sensitivity (at the quantum limit) of $1 \mathrm{~nm}$ for a single shot measurement in a bandwidth of $1.3 \mathrm{MHz}$ [14].

Finally, a 2D $(\mathrm{x}, \mathrm{z})$ experimental image of a phantom composed of two polyamide tubes with a an internal diameter of $800 \mu \mathrm{m}$ filled with red ink and submerged at $-3.5 \mathrm{~mm}$ depth in a tank filled with water has been reconstructed from a 1D LOFI temporal acquisition along the water surface.

Starting from 18 displacement measurements with an average SNR of 10 , the reconstructed image exhibits a SNR of the order of 200 in agreement with the theoretical prediction. The reconstituted width of the tube section is of the order of $1 \mathrm{~mm}$ is higher than the real one (i.e. $>$ $800 \mu \mathrm{m})$. This value can be explained, principally by the numerical aperture defined by of the measurement locations. 
In the context of PA imaging for biomedical applications, and by comparison with the widely used PZT detection, the LOFI sensor displays the following advantages for the detection of laser-induced ultrasonic signal: The LOFI setup is a self-aligned interferometer which allows non-contact optical measurements is a very simple way. Therefore, with a LOFI setup, there is a priori no technical difficulties in integrating PA imaging with conventional other imaging modalities. Also, with a LOFI setup offers the possibility to control the size of the detection area and therefore of the numerical aperture. So whatever the imaging depth, we can adapt the detection range to obtain the same values for both the lateral and transversal resolutions. Also, with its high sensitivity to low optical Feedback the LOFI setup allows to respect the MPE condition for biomedical applications.

In return, the main disadvantages of the LOFI setup are: The equivalent overpressure sensitivity of the LOFI setup $(\approx 1 \mathrm{kPa}$ for a single-shot measurement) is worse than the one of a usual PZT detection (of the order of $10 \mathrm{~Pa}$ ) for an equivalent detection bandwidth [20,21]. The time delay to acquire the ultrasonic signal is long due to the fact that the LOFI setup is a punctual detector. Even if the frequency detection bandwidth of an optical detection can be higher than the one of a resonant PZT sensor, in the demonstration shown here the LOFI detection bandwidth is in the megahertz range.

Actually, the use of $100 \mathrm{MHz}$ bandwidth with a slightly modified LOFI setup with a higher carrier frequency is under investigation. Also, the use of a LOFI setup to realize in vivo PAT imaging of the vascular network in mouse ear is currently under progress with our PA setup [22] where chirped signal with frequency sweep modulation need to be used to recover ( by the way of a correlation processing) the time delays between the acoustic sources and the detection locations [5]. This process requires the use of a much larger detection bandwidth than $1 \mathrm{MHz}$ to obtain a good resolution.

Also actually, we develop a new LOFI setup for fast PAT imaging where the output laser beam is divided into 3 laser beams which for each specific frequency shifter. In this set up, each beams is focused at a specific spatial position on the surface under investigation $[10,23]$. We hope that the simultaneous demodulation of the LOFI signal at the different specific frequencies allows to measures simultaneously the surface displacements at different locations and therefore to allow a fast reconstruction of the acoustic sources imaging without any scanning of the laser beams on the detection surface.

Funding Information. Université Grenoble Alpes (UGA): "AGIR" program; Centre National de la Recherche Scientifique (CNRS): "DEFI" program.

\section{REFERENCES}

1. L.V. Wang, Photoacoustic Imaging and Spectroscopy (CRC Press, 2009)

2. C. Li and L.V. Wang, "Photoacoustic tomography and sensing in biomedicine," Phys. Med. Biol. 54, R54-R97 (2009)

3. P. Beard, "Biomedical photoacoustics imaging," Interface focus 1,602$631(2011)$

4. S. ManoHar, and D. Razansky, "Photoacoustics: a historical review," Advances in Optics and Photonics 8,586-617 (2016)

5. S. Telenkov, and A. Mandelis, "Signal to noise analysis of biomedical photoacoustic measurements in time and frequency domain," Rev. Sci. Instrum. 81, 124901(2010)
6. O. Ogunlade, J.O.Y. Ho, T. L. Kalber, R. E. Hynds, E. Zhang, S. M. Janes, M. A. Birchall, C. R. Butler, and P. Beard, “Monitoring neovascularization and integration of decellularized human scaffolds using photoacoustic imaging," Photoacoustics 13, 76-84 (2019)

7. L.V. Wang, and S. Hu," Photoacoustic Tomography: In Vivo Imaging from Organelles to Organs," Sciences 335,1458-1462 (2012)

8. B. Dong, C. Sun and H. F. Zhang, "Optical detection of ultrasound in photoacoustic imaging," IEEE Trans. Biomed. Engi. 54, 4-15 (2017)

9. T. Taimre, M. Nikolic, K. Bertling, Y. L. Lim, T. Bosch and A. D. Rakic,"'Laser feedback interferometry: a tutorial on the self-mixing effect for coherent sensing," Adv. in Opt. and Photon. 7, 570,631 (2015)

10. K. Otsuka, "Self-Mixing Thin-Slice Solids-State Laser Metrology," Sensors 11, 2195-2245 (2011).

11. Y. Tan, S. Zhang, S. Zhang, Y. Zhang and N. Liu, "Response of microchip solid state laser to external frequency-shifted feedback and its applications," Sci. Rep. 3, 2912 (2013).

12. K. Otsuka, K. Abe, J.Y. Ko, and T.S. Lim, "Real-time nanometer vibration measurement with self-mixing microchip solid-state laser," Opt. Lett. 27, 1339-1341 (2002).

13. V. Giradeau, C. Goloni, O. Jacquin, O. Hugon, M. Inglebert, E. Lacot, "Nonlinear laser dynamics induced by frequency shifted optical feedback : application to vibration measurements," Appl. Opt. 55, 4049-4055 (2016).

14. V. Giradeau, O. Jacquin, O. Hugon, and E. Lacot, "Ultrasound vibration measurements based on laser optical feedback imaging," Appl. Opt. 57, 7634-7643 (2018)

15. E. Lacot, O. Jacquin, G. Roussely, O. Hugon, H. Guillet de Chatellus, "Comparative study of autodyne and heterodyne laser interferometry for imaging," J. Opt. Soc. Am. A 27, 2450-2458 (2010).

16. O. Jacquin, E. Lacot, W. Glastre, O. Hugon, H. Guillet de Chatellus, "Experimental comparison of autodyne and heterodyne laser interferometry using an Nd:YVO4 microchip laser," J. Opt. Soc. Am. A 28, 1741-1746 (2011).

17. S. A. Carp and V. Venugopalan,"Optoacoustic imaging based on the interferometric measurement of surface displacement," J. of Biomed. Opt. 12, 064001 (2007).

18. C. K. Liao, M. L. Li, and P. C. Li, "Optoacoustic imaging with synthetic aperture focusing and coherence weighting," Opt. Lett. 29, 2506-2508 (2004)

19. American National Standard for Safe Use of Lasers, Laser Inst. Amer., ANSI Standard Z136.1-2000, NY (2000)

20. J. Yao, L.V. Wang, "Sensitivity of photoacoustic microscopy," Photoacoutics 2,87-101 (2014)

21. A.M. Winkler, K. Maslov, L.V. Wang, "Noise-equivalent sensitivity of photoacoustics," J. Biomed. Opt. 18, 097003 (2013)

22. O. Hugon, B. Van Der Sanden, M. Inglebert, O. Jacquin, C. Misbah and E. Lacot,"Multi-wavelength photo-acoustic microscopy in frequency domain for simultaneous excition and detection of dyes,"Biomed Opt. Express 10, 932-943 (2019)

23. K. Otsuka, K. Abe, S. Sano, S. Sudo, J.Y. Ko, "Two-channel selfmixing laser Doppler measurement with carrier frequency-divisionmultiplexing," Appl. Opt. 44, 1709-1714 (2005). 